\title{
Positive undecidable numberings in the Ershov hierarchy*
}

\author{
Mustafa Manat ${ }^{\dagger}$ and Andrea Sorbi ${ }^{\ddagger}$
}

\begin{abstract}
We give a sufficient condition for an infinite computable family of $\Sigma_{a}^{-1}$ sets, to have computable positive but undecidable numberings, where $a$ is a notation for a nonzero computable ordinal. This extends a theorem proved by Talasbaeva for the finite levels of the Ershov hierarchy. In particular the family of all $\Sigma_{a}^{-1}$ sets has positive undecidable numberings: this verifies for all levels of the Ershov hierarchy a conjecture due to Badaev and Goncharov. We point out also that for every ordinal notation $a$ of a nonzero ordinal, there are families of $\Sigma_{a}^{-1}$ sets having positive numberings, but no Friedberg numberings: this answers for all levels (whether finite or infinite) of the Ershov hierarchy, a question originally raised, only for the finite levels over level 1, by Badaev and Goncharov.
\end{abstract}

\section{Introduction}

The results of Talasbaeva's paper [9] and of this paper are partly motivated by the observation that for the arithmetical hierarchy, questions about the existence of Friedberg numberings for a family may be reduced to the existence of positive numberings. Indeed, Goncharov and Sorbi [6] show that if a family of $\Sigma_{n}^{0}$ sets, $n \geq 2$, has positive numberings then it has Friedberg numberings as well. A natural problem is to see to what extent this, or similar circumstances, carry over to the Ershov hierarchy. It is shown in [9] that for every finite level $n$ of the Ershov hierarchy, every infinite family containing $\emptyset$ if $n$ is even, or $\omega$ if $n$ is odd, has infinitely many positive undecidable numberings, which are pairwise incomparable with respect to Rogers reducibility of numberings. (For $n=1$ this was first proved by Badaev [1].) We prove something similar for all levels $\Sigma_{a}^{-1}$ of the Ershov hierarchy, where $a$ is the ordinal notation of any nonzero

* Part of the research contained in this paper was carried out while the first author was visiting the Department of Mathematics and Computer Science "Roberto Magari" of the University of Siena, Italy. The first author wishes to thank the Al-Farabi University for supporting the visit. The authors thank Prof. Serikzhan A. Badaev for precious help and useful comments.

${ }^{\dagger}$ Kazakh National University, Al-Farabi ave., 71, Almaty, 050038, Kazakhstan

${ }^{\ddagger}$ Dipartimento di Scienze Matematiche ed Informatiche "Roberto Magari", Università di Siena, 53100 Siena, Italy 
computable ordinal: in particular we show that if $a$ is notation of an infinite computable ordinal, and $\mathcal{A}$ is an infinite family of $\Sigma_{a}^{-1}$ sets, containing some set $A$ which belongs to some finite level of the Ershov hierarchy, then $\mathcal{A}$ has infinitely many positive undecidable numberings, which are pairwise incomparable with respect to Rogers reducibility. As a consequence, the family of all $\Sigma_{a}^{-1}$ sets has positive undecidable numberings, verifying Conjecture 15 of [2] for all levels of the Ershov hierarchy. (Of course, for finite levels this conjecture had been verified by Talasbaeva's theorem). A straightforward observation, derived as a consequence of Ospichev's theorem on the existence, at all levels, of families without Friedberg numberings, allows us to show also that at every level there exist families with positive numberings but without Friedberg numberings, answering negatively Question 17 of [2].

We refer to Kleene's system $O$ of ordinal notations for computable ordinals, as presented in [8]. If $a \in O$, then the symbol $|a|_{O}$ indicates the ordinal denoted by $a$. We begin by recalling the definition of the Ershov hierarchy, $[3,4,5]$. Our characterization below is due to Ospichev [7].

Definition 1.1. If $a$ is a notation for a computable ordinal, then a set of numbers $A$ is said to be $\Sigma_{a}^{-1}$ (or $A \in \Sigma_{a}^{-1}$ ) if there are a computable function $f(z, t)$ and a partial computable function $\gamma(z, t)$ such that, for all $z$,

1. $A(z)=\lim _{t} f(z, t)$, with $f(z, 0)=0$; (here and in the following, for a given set $X, X(x)$ denotes the value of the characteristic function of $X$ on $x$;)

2. $\gamma(z, t) \downarrow \Rightarrow \gamma(z, t+1) \downarrow$, and $\gamma(z, t+1) \leq_{O} \gamma(z, t)<_{O} a$;

3. $f(z, t+1) \neq f(z, t) \Rightarrow \gamma(z, t+1) \downarrow \neq \gamma(z, t)$.

We call the partial function $\gamma$ the mind-change function for $A$, relatively to $f$.

A $\Sigma_{a}^{-1}$-approximation to a $\Sigma_{a}^{-1}$-set $A$, is a pair $\langle f, \gamma\rangle$, where $f$ and $\gamma$ are respectively a computable function and a partial computable function satisfying $1-3$, above, for $A$.

If the ordinal $|a|_{O}=n$ is finite, we also write $\Sigma_{n}^{-1}$ instead of $\Sigma_{a}^{-1}$, as notations for finite ordinals are unique.

Following the general approach to the theory of numberings proposed by [6], we can give the following definition:

Definition 1.2. A $\Sigma_{a}^{-1}$-computable numbering, or simply a computable numbering, of a family $\mathcal{A}$ of $\Sigma_{a}^{-1}$-sets is an onto function $\pi: \omega \longrightarrow \mathcal{A}$, such that the set

$$
\{\langle k, x\rangle: x \in \pi(k)\} \in \Sigma_{a}^{-1} .
$$

Therefore it is easy to see that a computable numbering of a family $\mathcal{A}$ of $\Sigma_{a}^{-1}$-sets is an onto function $\pi: \omega \longrightarrow \mathcal{A}$ for which there exist a computable function $f(k, x, t)$ and a partial computable function $\gamma(k, x, t)$, such that for all $k, x, t$,

1. $\pi(k)(x)=\lim _{t} f(k, x, t)$, with $f(k, x, 0)=0$; 


$$
\begin{aligned}
& \text { 2. } \gamma(k, x, t) \downarrow \Rightarrow \gamma(k, x, t+1) \downarrow ; \gamma(k, x, t+1) \leq_{O} \gamma(k, x, t)<_{O} a \text {; and } \\
& \text { 3. } f(k, x, t+1) \neq f(k, x, t) \Rightarrow \gamma(k, x, t+1) \downarrow \neq \gamma(k, x, t) .
\end{aligned}
$$

We recall (see e.g. [7]) that there is an effective indexing $\left\{\nu_{e}\right\}_{e \in \omega}$ of all computable numberings of families of $\Sigma_{a}^{-1}$ sets, i.e. an indexing satisfying

$$
\left\{\langle e, k, x\rangle: x \in \nu_{e}(k)\right\} \in \Sigma_{a}^{-1}
$$

and from $e$ one has (see [7]) an effective way of getting a computable function $f_{e}$ and a partial computable function $\gamma_{e}$ witnessing that the set $\left\{\langle k, x\rangle: x \in \nu_{e}(k)\right\}$ is $\Sigma_{a}^{-1}$, as in Definition 1.2.

We will write $\operatorname{Com}_{a}^{-1}(\mathcal{A})$ to denote the set of computable numberings of a family $\mathcal{A} \in \Sigma_{a}^{-1}$. A family $\mathcal{A} \in \Sigma_{a}^{-1}$ is computable if $\operatorname{Com}_{a}^{-1}(\mathcal{A}) \neq \emptyset$. If $\alpha, \beta$ are numberings of a same family, let $\alpha \leq \beta$ if there is a computable function $f$ such that $\alpha=\beta \circ f$. The relation $\leq$ is a reducibility (called Rogers reducibility), and gives rise to a degree structure, where a degree (called a Rogers degree) is the equivalence class of a numbering under the equivalence relation $\equiv$ generated by $\leq$ : the set of Rogers degrees of the elements in $\operatorname{Com}_{a}^{-1}(\mathcal{A})$ is denoted by $\mathfrak{R}_{a}^{-1}(\mathcal{A})$, and called the Rogers semilattice of $\mathcal{A}$ : it is well known that if $\mathfrak{R}_{a}^{-1}(\mathcal{A}) \neq \emptyset$ then $\mathfrak{R}_{a}^{-1}(\mathcal{A})$ is an upper semilattice. An infinite subset $X \subseteq \mathfrak{R}_{a}^{-1}(\mathcal{A})$ is an antichain if for every pair of Rogers degrees $\mathbf{a}, \mathbf{b} \in X$ we have $\mathbf{a} \not \leq \mathbf{b}$ and $\mathbf{b} \not \leq \mathbf{a}$.

Definition 1.3. Let $\alpha$ be a numbering of a family $\mathcal{A}$. Then $\alpha$ is called a Friedberg numbering, if $\alpha(i) \neq \alpha(j)$ for every $i \neq j$; $\alpha$ is called decidable if $\{\langle i, j\rangle: \alpha(i)=\alpha(j)\}$ is a decidable set; $\alpha$ is positive if $\{\langle i, j\rangle: \alpha(i)=\alpha(j)\}$ is a computably enumerable (c.e.) set.

Of course, if $\alpha$ is a Friedberg numbering, then $\alpha$ is decidable; and every decidable numbering is positive. Moreover, the following obvious and well known fact holds:

Lemma 1.4. If $\mathcal{A}$ is infinite, and $\alpha$ is a computable decidable numbering of $\mathcal{A}$, then $\mathcal{A}$ has a computable Friedberg numbering $\beta$ with $\alpha \equiv \beta$.

Proof. Let $\mathcal{A}$ be infinite, and suppose that $\alpha \in \operatorname{Com}_{a}^{-1}(\mathcal{A})$ is decidable. Then define $\beta \in \operatorname{Com}_{a}^{-1}(\mathcal{A})$ by:

- $\beta(0)=\alpha(0)$;

- suppose that $\beta(j)=\alpha\left(i_{j}\right)$, all $j \leq n$, and define $\beta(n+1)=\alpha\left(i_{n+1}\right)$, where $i_{n+1}$ is the least number $i$ such that $\alpha(i) \neq \alpha\left(i_{j}\right)$, for all $j \leq n$.

It follows that $\beta \in \operatorname{Com}_{a}^{-1}(\mathcal{A}), \beta$ is a Friedberg numbering, and $\beta \leq \alpha$. The converse reducibility $\alpha \leq \beta$ follows from the well known fact that the Rogers degree of every decidable (in fact, positive) numbering is minimal. 


\section{Positive numberings}

In this section we generalize to all levels of the Ershov hierarchy a theorem due to Talasbaeva, [9]:

Theorem 2.1 ([9]). For every $n \in \omega, n \geq 1$, and every infinite computable family $\mathcal{A} \subseteq \Sigma_{n}^{-1}$, if either

1. $n$ even and $\emptyset \in \mathcal{A}$, or

2. $n$ odd and $\omega \in \mathcal{A}$,

then $\operatorname{Com}_{a}^{-1}(\mathcal{A})$ contains infinitely many positive and undecidable numberings $\left\{\alpha_{i}\right\}_{i \in \omega}$, such that the corresponding Rogers degrees form an antichain.

By generalizing the argument in [9], we show that a similar results holds for the infinite levels of the Ershov hierarchy:

Theorem 2.2. Let $a \in O$ be a notation of an infinite ordinal. If $\mathcal{A} \subseteq \Sigma_{a}^{-1}$ is infinite, computable, and there exists $A \in \Sigma_{m}^{-1} \cap \mathcal{A}$ for some finite $m$, then $\mathrm{Com}_{a}^{-1}(\mathcal{A})$ contains infinitely many positive undecidable numberings $\left\{\alpha_{i}\right\}_{i \in \omega}$, such that the corresponding Rogers degrees form an antichain.

Proof. The proof is a straightforward generalization of [9]. Let $\mathcal{A}$ be an infinite family of $\Sigma_{a}^{-1}$ sets, where $a \in O$ is notation of an infinite ordinal, and let $\alpha \in \operatorname{Com}_{a}^{-1}(\mathcal{A})$, i.e. $\alpha$ is a computable numbering of $\mathcal{A}$. Let us assume that $\mathcal{A}$ contains some set $A \in \Sigma_{m}^{-1}$ for some finite $m$. Without loss of generality we assume that $\alpha(0)=A$. Also, let $f, \gamma$ be a total computable function and a partial computable function, respectively, witnessing that

$$
\{\langle n, x\rangle: x \in \alpha(n)\} \in \Sigma_{a}^{-1}
$$

as in Definition 1.2. We assume that the mind-change function $\gamma$ satisfies, for all $z$,

$$
\gamma(n, x, s) \downarrow \Rightarrow \gamma(n, x, s)>_{O} b,
$$

where $|b|_{O}=m$ : if this is not the case, then we define from $\gamma$ a new suitable mind-change function $\gamma^{\prime}$ with this property, by letting $\gamma^{\prime}(n, x, s)=\gamma(n, x, s)$ if $|\gamma(n, x, s)|_{O}$ not finite or $\gamma(n, x, s) \uparrow$, and letting $\gamma^{\prime}(n, x, s)=\gamma(n, x, s)+_{o} 2^{b}$ if $\gamma(n, x, s) \downarrow$ and $|\gamma(n, x, s)|_{O}$ is finite. Here $+_{O}$ is a partial computable function satisfying on $a, b \in O,\left|a+_{O} b\right|_{O}=|a|_{O}+|b|_{O}$ : hence, as $2^{b}$ is the notation for $m+1$, if $\gamma(n, x, s)$ is notation of a finite ordinal $k$, then $\left|\gamma^{\prime}(n, x, s)\right|_{O}=k+m+1$. It is easy to see that $\left\langle f, \gamma^{\prime}\right\rangle$ is still a $\Sigma_{a}^{-1}$-approximation to $\alpha$.

Let

$$
F=\{y>0:(\forall m<y)[\alpha(m) \neq \alpha(y)]\} .
$$

$F$ is evidently a $\Sigma_{2}^{0}$ set and thus we may assume that there is a uniformly computable sequence of finite sets $\left\{F_{s}\right\}_{s \in \omega}$ (meaning that the predicate " $x \in F_{s}$ " is decidable) such that $F(x)=\liminf _{s} F_{s}(x)$, i.e.

$$
F=\left\{y:(\exists t)(\forall s \geq t)\left[y \in F_{s}\right]\right\} .
$$


Requirements. The construction is by stages: at stage $t$ we will define the values of parameters $i(x, t)$ (one for each $i$ ), and we will set "restraints" on several numbers.

For each $i$ we will build a computable numbering $\alpha_{i}$ of $\mathcal{A}$, by simultaneously defining a computable function $f_{i}$ and a partial computable mind-change function $\gamma_{i}$ witnessing that $\alpha_{i} \in \Sigma_{a}^{-1}$, according to Definition 1.2. Eventually we will have that $\alpha_{i}(x)=\alpha(i(x))$, where $i(x)=\lim _{t} i(x, t)$ : in fact for every $i$ and $x, i(x, t)$ may change at most once, after being defined for the first time, and if $i(x, t) \downarrow \neq i(x, t+1)$ then for all $s \geq t+1, i(x, s)=0$.

We will achieve that $\alpha_{i}$ is positive by guaranteeing that the set

$$
W=\left\{e: \alpha_{i}(e)=\alpha(0)\right\}
$$

is c.e. (precisely: $x \in W$ if and only if there exists $t$ such that $i(x, t)=0$ ), and for every $y \in F$ there exists exactly one $z$ such that $\alpha_{i}(z)=\alpha(y)$. Hence,

$$
\alpha_{i}(x)=\alpha_{i}(y) \Leftrightarrow[x=y \text { or } x, y \in W] .
$$

Additionally, the construction aims at satisfying the following requirements, for all $i, j, k, y$, with $i \neq j$ :

$$
\begin{aligned}
P_{i, k}: & \left\{e: \alpha_{i}(e) \neq \alpha(0)\right\} \neq W_{k}, \\
Q_{i, y}: & y \in F \Rightarrow(\exists ! z)\left[\alpha_{i}(z)=\alpha(y)\right], \\
R_{i, j, k}: & \varphi_{k} \text { total } \Rightarrow \alpha_{j} \neq \alpha_{i} \circ \varphi_{k} .
\end{aligned}
$$

Indeed, satisfaction of all $P_{i, k}$ ensures that $\left\{e: \alpha_{i}(e) \neq \alpha(0)\right\}$ is not c.e., hence the numbering $\alpha_{i}$ is not decidable. Satisfaction of all $R_{i, j, k}$ ensures that if $i \neq j$ then $\alpha_{j} \not \leq \alpha_{i}$. Finally, satisfaction of all $Q_{i, y}$ ensures that, for every $i$, $\alpha_{i}$ is a numbering of the family $\mathcal{A}$ (and $\alpha_{i}$ is positive, as already observed). A routine updating procedure performed at the end of each stage will guarantee that for every $i, z$, the value $i(z)$ is eventually defined.

Strategies to satisfy the requirements. We briefly outline the strategies to satisfy the requirements in isolation.

1. (Module for $\left.P_{i, k}\right)$ : wait for an element $x \in W_{k}$, and let $i(x)=0$, so that $\alpha_{i}(x)=\alpha(0)$ and thus $x \in W_{k}-\left\{e: \alpha_{i}(e) \neq \alpha(0)\right\}$. The construction will guarantee that if $W_{k}$ is infinite, then one can indeed find such an $x$; on the other hand, since $\alpha_{i}$ is a numbering of $\mathcal{A}$, which is infinite, we have that $\left\{e: \alpha_{i}(e) \neq \alpha(0)\right\}$ is infinite: hence, for every $k,\left\{e: \alpha_{i}(e) \neq \alpha(0)\right\} \neq W_{k}$.

2. (Module for $Q_{i, y}$ ): if $y \in F$ and there is as yet no $z$ such that $i(z)=y$, then pick a suitable targeted $z$ and define $i(z)=y$. If $i(z)=y$ is already defined, but now $y \notin F$ (due to the way $F$ is approximated as a $\Sigma_{2}^{0}$ set), then respond by letting $i(z)=0$.

3. (Module for $R_{i, j, k}$ ): appoint a witness $z$, with $j(z)=y$, for a suitable $y \in F$; restrain $j(z)=y$ and wait for $\varphi_{k}(z) \downarrow=x$; then let $i(x)=0$. Thus $\alpha_{i}\left(\varphi_{k}(z)\right)=\alpha(0)$, and $\alpha_{j}(z)=\alpha(y)$, winning the requirement since $\alpha(y) \neq \alpha(0)$. 
The priority listing of requirements. We list the requirements according to the following priority listing:

$$
P_{0}<Q_{0}<R_{0}<P_{1}<Q_{1}<R_{1}<\cdots<P_{i}<Q_{i}<R_{i} \cdots .
$$

(For a $P$-requirement, or a $Q$-requirement, the subscript codes a pair of numbers, whereas for an $R$-requirement the subscript codes a triple of numbers: we refer here to suitable computable codings of pairs and triples of numbers.)

We anticipate that $Q_{i, r}$ does not refer to a number $r \in F$, but rather to the $r$-th element $y(r)$ that gets stabilized in the $\Sigma_{2}^{0}$-approximation to $F$, in the sense of Lemma 2.4: in other words we act for $Q_{i, r}$ as previously described for $Q_{i, y(r)}$. The turn from $r$ to $y(r)$ is meant to cope with the fact that $r$ may not get stabilized in the $\Sigma_{2}^{0}$ approximation of $F$ (if $r \notin F$ ), but $y(r)$ always does.

For $N, N^{\prime}$ in this list, if $N<N^{\prime}$ we also say that $N$ has higher priority than $N^{\prime}$ (or $N^{\prime}$ has lower priority than $N$.) Sometimes we also talk about the priority rank of the various strategies, meaning that a strategy for a requirement $N$ has higher priority than a strategy for a requirement $N^{\prime}$, if $N$ has higher priority than $N^{\prime}$. In fact, we often identify a requirement with its strategy. At each stage we look for the least requirement $N$ which requires attention (according to a precise definition which will be given later in the construction), and we act to satisfy $N$ following the strategy outlined above: our only concern will be that our action does not injure any of the higher priority strategies. If our action on $N$ injures a strategy for a lower priority requirement $N^{\prime}$, then $N^{\prime}$ will have to be reconsidered again.

We spend a few words on how we avoid strategies for $R$-requirements to interfere with higher priority strategies. We will see in the construction that in order to act, $Q$-strategies and $R$-strategies need to restrain some definitions of the form $j(z)=y$, with $y \in F$ : let us say in this case that the strategy restrains $j(z)=y$ (or simply $j(z) \in F$, if it does not matter to specify the particular $y \in F$ ). Consider now the requirement $N=R_{i, j, k}$, and let us call $\sigma$ the strategy acting for $N$. Let $X$ be the finite set consisting of the numbers $x$ such that $i(x) \in F$ is restrained by some higher priority strategy: thus $\sigma$ can not define $i(x)=0$ for any $x \in X$. A naive strategy here would be to wait for $\varphi_{k}$ to converge on some $z$ to some $x \notin X$ (if range $\left(\varphi_{k}\right) \subseteq X$, then $N$ is satisfied, since $\varphi_{k}$ can not reduce $\alpha_{j}$ to $\alpha_{i}$, which are both numberings of $\mathcal{A}$, which is infinite), and to restrain $j(z)=y$, for some $y \in F$. This approach has the potential danger that we may come up with some $z$ for which the construction has already provided the definition $j(z)=0$ : so we must appoint a witness $z$ first, restrain $j(z)=y$ for some $y \in F$, wait for $\varphi_{k}(z)$ to converge to some $x$, act as before if $x \notin X$; on the other hand if $x \in X$, we repeat the procedure, by picking a new $z$ and a different $y \in F$. One suitable way to implement this idea (without even appointing the witnesses one by one, but appointing, once for all, sufficiently many of them) is the following: Let $m=|X|$ be the number of elements of $X$ : then $\sigma$ appoints $m+1$ new pairwise different numbers $z_{0}, \ldots, z_{m}$ with new injective definitions $j\left(z_{u}\right)=y_{u}, y_{u} \in F$, and relative restraints; subsequently, $\sigma$ waits for $\varphi_{k}$ to converge on all these numbers $z_{u}$, say $\varphi_{k}\left(z_{u}\right)=x_{u}$ (otherwise 
$\varphi_{k}$ is not total): since $\alpha_{j}\left(z_{u}\right)=\alpha\left(y_{u}\right)$ and thus $\alpha_{j}\left(z_{u}\right) \neq \alpha_{j}\left(z_{u^{\prime}}\right)$ for $u \neq u^{\prime}$, if $\varphi_{k}$ is a reduction of $\alpha_{j}$ to $\alpha_{i}$, then by cardinality it must be the case that there exists $u$ such that $x_{u} \notin X$ : for this $x_{u}$, we are thus able to define $i\left(x_{u}\right)=0$.

On the other hand, suppose that some $Q$ - or $R$-strategy defines $j(z)=0$ : a lower priority $R$-requirement $R_{i, j, k}$ is injured if it currently has a restraint of the form $j(z) \in F$, and so we loose for this requirement the possibility that $\alpha_{j}(z) \neq \alpha(0)$. In this case $R_{i, j, k}$ sets up new restraints, relying on the fact that it can be so injured only finitely many times. Similarly the move $j(z)=0$ may injure some $Q_{i, r}$ of lower priority requirement with current restraint $j(z)=y(r)$ : in this case we redefine $j\left(z^{\prime}\right)=y(r)$, for a new $z^{\prime}$. Again we rely on the fact that $Q_{i, r}$ may be so injured only finitely many times.

The construction. The construction is by stages. It is understood below that in going from stage $s$ to stage $s+1$, any parameter which is not explicitly redefined will maintain the same value (if any) possessed at stage $s$. Similarly any restraint imposed by some strategy at stage $s$ is still valid at stage $s+1$, unless the restraint is explicitly dropped at $s+1$. We say that a number is new at $s$ if it bigger than all numbers mentioned so far in the construction.

At stage $s$, for each $y \in F_{s}$, say that $t \leq s$ is the age of $y$, if $t$ is the smallest stage $\leq s$ such that

$$
(\forall u)\left[t \leq u \leq s \Rightarrow y \in F_{u}\right] .
$$

Define

$$
L(s)=\left\{\langle t, y\rangle: y \in F_{s} \text { and } t \text { age of } y\right\},
$$

and let $c(s)$ be the cardinality of $L(s)$. Let $w(r, s)$ denote the $r$-th element of $L(s)$ in order of magnitude (of course $r<c(s)$ ), and let also $t(r, s), y(r, s)$ be such that

$$
w(r, s)=\langle t(r, s), y(r, s)\rangle .
$$

Definition 2.3. We say that $L(s)$ breaks before $r$ at $s+1$ if $r \geq c(s+1)$, or there exists $r^{\prime} \leq r$ such that $w\left(r^{\prime}, s\right) \notin L(s+1)$.

Lemma 2.4. For every $s$, we have $y \in F_{s}$ if and only if there exists exactly one $r<c(s)$ such that $y=y(r, s)$. Moreover, for every $r, \lim _{s} w(r, s), \lim _{s} t(r, s)$ $\lim _{s} y(r, s)$ exist, and $F=\{y(r): r \in \omega\}$, where $y(r)=\lim _{s} y(r, s)$. In particular, for every $r$, there is a stage $t$ such that at no $s \geq t$ does $L(s)$ breaks before $r$ at $s$.

Proof. The proof is a straightforward consequence of the fact that $F$ is a $\Sigma_{2}^{0}$ set and of (2.1). The proof that $\lim _{s} w(r, s)$ exists, is by induction on $r$.

Stage 0 . For every $i$, define $i(0,0)=0$. (Since $i(0, t)$ will not change any more, this permanently makes $\alpha_{i}(0)=\alpha(0)$.) All other $i(z, 0)$ are undefined. No strategy is setting any restraint at stage 0 .

For every $i, u$, let $f_{i}(0, u, 0)=f(0, u, 0), \gamma_{i}(0, u, 0)=\gamma(0, u, 0)$; and for every $x>0, u$, let $f_{i}(x, u, 0)=0$ and $\gamma_{i}(x, u, 0)=\uparrow$. 
Stage $s+1$. We begin with the definition of requiring attention at $s+1$ :

1. If $N=P_{i, k}$ then we say that $N$ requires attention if there is no $u \in W_{k, s}$ such that $i(u, s)=0$, but there is $x \in W_{k, s}$ such that $i(x, s) \in F$ is not restrained by higher priority strategies.

2. If $N=Q_{i, r}$, then we say that $N$ requires attention if either

(a) $N$ is currently setting no restraint; or

(b) $L(s)$ breaks before $r$ at $s+1$.

3. If $N=R_{i, j, k}$ then we say that $N$ requires attention if either

(a) $N$ is currently setting no restraint; or

(b) $N$ is restraining $j\left(z_{0}, s\right)=y(l, s), \ldots, j\left(z_{m}, s\right)=y(l+m, s)$, (where the parameters $m=m(N, s)$ and $l(N, s)$ are defined in the construction), and $L(s)$ breaks before $l+m$ at $s+1$; or

(c) as before $N$ is restraining $j\left(z_{0}, s\right)=y(l, s), \ldots, j\left(z_{m}, s\right)=y(l+m, s)$, $\varphi_{k, s}$ is totally defined and injective on the set $\left\{z_{0}, z_{1}, \ldots, z_{m}\right\}$, for no $u \leq m$ we have $\varphi_{k, s}\left(z_{u}\right)=y$ with $i(y, s)=0$, and there is some $u \leq m$ such that $\varphi_{k, s}\left(z_{u}\right)=x$, and no higher priority strategy is restraining $i(x, s) \in F$.

If there is no $P_{u}-$ requirement, or $Q_{u}-$ requirement, or $R_{u}-$ requirement that requires attention, any $u \leq s$, then end the stage. Otherwise, let $N$ be the least requirement, which requires attention. We distinguish three cases.

1. If $N=P_{i, k}$ and $x$ is the least number as in the definition of requiring attention, then let $i(x, s+1)=0$. End the stage, as specified below, in the procedure "End of stage".

2. If $N=Q_{i, r}$ then we act correspondingly to the various clauses for requiring attentions:

(a) pick a new $z$, let $i(z, s+1)=y(r, s+1)$, restrain $i(z, s+1)=y(r, s+1)$;

(b) drop any restraint for $N$; if $i(z, s) \in F$ was the current restraint, then let $i(z, s+1)=0$.

End the stage.

3. If $N=R_{i, j, k}$ then we act correspondingly to the various clauses for requiring attentions:

(a) let $m=m(N, s+1)$ be the cardinality of the set

$$
X(N, s+1)=\left\{x:\left(\exists N^{\prime}<N\right)\left[N^{\prime} \text { is restraining } i(x, s) \in F\right]\right\} ;
$$

let $l=l(N, s+1)$ be the least element (if any; $l(N, s+1)=-1$, otherwise) such that, for every $N^{\prime}<N$ if $N^{\prime}$ is restraining $j^{\prime}\left(z^{\prime}, s\right)=$ 
$y^{\prime}$ then $y^{\prime}=y(r, s)$, for some $r<l$; pick new numbers $z_{0}, \ldots, z_{m}$, and for every $u \leq m$ define and restrain (with priority that of $N$ )

$$
j\left(z_{u}, s+1\right)=y(l+u, s+1) ;
$$

(b) drop any restraint for $N$ : for any previous restraint $j(z, s) \in F$ set by $N$, let $j(z, s+1)=0$.

(c) pick the least $x$ as in the definition of requiring attention, and let $i(x, s+1)=0$.

End the stage.

Procedure "End of stage". At the end of the stage we perform additional tasks:

1. Resetting. If we end stage $s+1$ due to the action of a strategy on behalf of a requirement $N$, then we reset all $N^{\prime}$ of lower priority $\left(N<N^{\prime}\right)$, where $N^{\prime}$ is a $Q$-or an $R$-requirement: if $N^{\prime}$ restrains $j(z, s) \in F$, then we drop the restraint, and we define $j(z, s+1)=0$.

2. Updating the definition of $j(z)$. For every $j$, and every $z \leq s$, if $j(z, s+1)$ is undefined, then let $j(z, s+1)=0$.

3. Updating the $\Sigma_{a}^{-1}$-approximations. For each $j, z$ such that $j(z, s) \neq 0$ (including $j(z, s)$ undefined), but we define $j(z, s+1)=0$, we update $f_{j}$ by letting, for every $u, f_{j}(z, u, s+1)=f(0, u, s+1)$, and $\gamma_{j}(z, u, s+1)=b$ (since so far either $\gamma_{j}(z, u, t)$ was undefined, or $\gamma_{j}(z, u, t)$ has been defined through values of $\gamma$ and thus $>_{O} b$, this is a safe definition of the mindchange function).

If already $j(z, s)=0$, then for every $u$ define $f_{j}(z, u, s+1)=f(0, u, s+1)$, and if $f_{j}(z, u, s+1) \neq f_{j}(z, u, s)$ then let $\gamma_{j}(z, u, s+1)=c$ where $c$ is the ordinal notation for the predecessor of $\left|\gamma_{j}(z, u, s)\right|_{O}$, which is a finite ordinal, by the previous item.

In all other cases: if $j(z, s+1)=y$, then let $f_{j}(z, u, s+1)=f(y, u, s+1)$ and $\gamma_{j}(z, u, s+1)=\gamma(y, u, s+1)$; if $j(z, s+1)$ is undefined, then let $f_{j}(z, u, s+1)=0$ and $\gamma_{j}(z, u, s+1)=\uparrow$.

Verification. The verification is based upon the following lemmas:

Lemma 2.5. Every requirement requires attention only finitely often. For every requirement $N$, if $Y(N, s)$ is the set of pairs $(z, y)$ such that $N$ restrains at $s$ the definition $j(z, s)=y(r, s)$, for some $r$ and a fixed $j$ depending on $N$, then there exists a stage $t$ such that $Y(N, s)=Y(N, t)$, for every $s \geq t$. There was confusion in notations: see the last paragraph on page 8

Proof. Assume that $t_{0}$ is the least stage at which no requirement $N^{\prime}<N$ requires attention. So at every $s>t_{0}$, no $N^{\prime}<N$ requires attention, nor does it end the stage. 
If $N$ is a $P$-requirement, $N=P_{i, k}$, then $N$ may require attention at most once after $t_{0}$, at the least stage $s+1>t_{0}$ (if any) at which we find some $x \in W_{k, s}$ such that $i(x, s) \in F$ is not restrained by any higher priority strategy, and on the other hand there is no $u \in W_{k, s}$ such that already $i(u, s)=0$.

If $N=Q_{i, r}$, by Lemma 2.4 let $t_{1} \geq t_{0}+1$ be the least stage such that $L(s)$ does not break before $r$ at any $s \geq t_{1}$ : so $y(r, s)=y\left(r, t_{1}\right)=y(r)$. Then either $N$ does not require attention at $t_{1}$ as there is already $z$ such that $i\left(z, t_{1}\right)=y(r)$. (Notice that for all $s \geq t_{1}, i(z, s)=y(r)$, since the definition $i(z, s)=y(r)$ is restrained by some higher priority strategy.) Or, at $t_{1}$ we pick a new $z$ and define $i\left(z, t_{1}\right)=y(r)$, with restraint that of $N$ : this values will not change any more, so $N$ will not require attention at any future stage.

If $N=R_{i, j, k}$, then for every $s>t_{0}, m(N, s)=m\left(N, t_{0}+1\right)=m$, and $l(N, s)=l\left(N, t_{0}+1\right)=l$. By Lemma 2.4 let $t_{1} \geq t_{0}+1$ be the least stage such that $L(s)$ does not break before $l+m$ at any $s \geq t_{1}$ : so for every $r \leq l+m$, $y(r, s)=y\left(r, t_{1}\right)=y(r)$. So at stage $t_{1}$ we appoint for the last time $z_{0}, \ldots, z_{m}$, and define $j\left(z_{0}\right)=y(l), \ldots, j\left(z_{m}\right)=y(l+m)$, with restraint that of $N$. After this, $N$ may require attention at most once, upon finding at some further stage $s, x$ such that $\varphi_{k, s}\left(z_{u}\right)=x$ for some $z_{u}$, and $i(x, s) \in F$ not restrained by any higher priority strategy.

Next we notice that $i(z)=\lim _{s} i(z, s)$ exists for every $z$ : this follows trivially by item 2 . of the procedure "end of stage".

We say that $Q_{i, r}$ is satisfied if there exists exactly one $z>0$ such that $i(z)=y(r)$. We say that $P_{i, k}$ is satisfied if $\left\{e: \alpha_{i}(e) \neq \alpha(0)\right\} \neq W_{k}$. Finally we say that $R_{i, j, k}$ is satisfied $(i \neq j)$ if $\varphi_{k}$ is not total or $\alpha_{j} \neq \alpha_{i} \circ \varphi_{k}$. This permutation is caused by a problem of hyphenation in Russian text

Lemma 2.6. For every $i, j, k, r(i \neq j), Q_{i, r}, P_{i, k}$, and $R_{i, j, k}$ are satisfied. (This also implies that $\alpha_{i}$ is a numbering of the whole family).

Proof. Let $i, r$ be given. By the previous lemma there is a last stage $t$ at which $Q_{i, r}$ requires attention. At $t$ either there is already some definition $i(z)=y(r)$ (where $y(r)$ is the limit value of $y(r, s)$ ), restrained by some higher priority strategy, or we define once and for all $i(z)=y(r)$ for a new $z$. Notice also that by item 1 . of the procedure "end of stage", and by the fact that when we drop a restraint $j(u) \in F$ we define $j(u)=0$, for no $y \in F$ there can be more than one $z$ such that $i(z)=y$.

For $P_{i, k}$ notice that if $P_{i, k}$ at some stage requires attention then $P_{i, k}$ is trivially satisfied at that stage. Otherwise, $W_{k}$ is finite, but in this case $P_{i, k}$ is satisfied, since by the previously proved item, the set $\left\{e: \alpha_{i}(e) \neq \alpha(0)\right\}$ is infinite.

For $N=R_{i, j, k}$, notice that by Lemma 2.5 there is a last stage $s_{0}$ at which we define the final value $Y(N)$ of $Y\left(N, s_{0}\right)$. Either $\varphi_{k}$ is undefined on some of the numbers $z$ such that there is a pair $(z, y) \in Y(N)$ with $j(z)=y$-but in this case $N$ is trivially met, since $\varphi_{k}$ is not total; or, by construction there are $m+1$ such numbers $z$, and $m$ numbers $x$ such that $i(x) \in F$ is restrained by higher priority strategies. Starting this point till the end of Lemma 2.6, I 
changed the original proof If $\varphi_{k}$ is not injective on these $z$, then there exist two pairs $(z, y),\left(z^{\prime}, y^{\prime}\right) \in Y(N)$ such that $\varphi_{k}(z)=\varphi_{k}\left(z^{\prime}\right)$ and $j(z) \neq j\left(z^{\prime}\right)$. Then $\alpha_{j} z=\alpha y \neq \alpha y^{\prime}=\alpha_{j} z^{\prime}$ and, therefore, $\varphi_{k}$ could not reduce numbering $\alpha_{j}$ to any numbering, in particular to $\alpha_{i}$. If $\varphi_{k}(z)=x$ and $i(x)=0$ for some number $x$ and pair $(z, y) \in Y(N)$ then $\alpha_{j}(z)=\alpha j(z) \neq \alpha 0$ and $\alpha_{i} \varphi_{k}(z)=\alpha_{i} x=\alpha 0$. Thus, in this case requirement $N$ is satisfied too. Finally, consider the case when $N$ requires attention on some stage $s+1 \geq s_{0}$. On stage $s+1$, there exist a pair $(z, y) \in Y(N)$ and a number $x$ such that $\varphi_{k, s}(z)=x$ and $i(x, s) \in F$ is not restrained by higher priority strategies. By construction, $i(x, s+1)=0$. Thus, $\alpha_{j} \neq \alpha_{i} \circ \varphi_{k}$, since $\alpha_{i} \varphi_{k}(z)=\alpha 0$ and $\alpha_{j} z=\alpha y \neq \alpha 0$.

Lemma 2.7. $\alpha_{i} \in \operatorname{Com}_{a}^{-1}(\mathcal{A})$.

Proof. It is enough to show that the pair $\left\langle f_{i}, \gamma_{i}\right\rangle$ is a $\Sigma_{a}^{-1}$-approximation to $\alpha_{i}$. For this notice that for $x>0, f_{i}(x, u, s)$ and $\gamma_{i}(x, u, s)$ are defined (at least from some stage on, before which $f_{i}(x, u, t)=0$ and $\gamma_{i}(x, u, t)$ is undefined) exactly as $f(y, u, s)$ and $\gamma(y, u, s)$, respectively (some $y)$ and while this happens the value of $\gamma_{i}(x, u, s)$, if defined, is $>_{O} b$; if and when we set $i(x, s)=0$, then after then we define $f_{i}(x, u, t)=f(0, u, t), \gamma_{i}(x, u, t)=b$ and, from now on, the changes of $\gamma_{i}(x, u, t)$ mirror the changes of $f(0, u, t)$, each time decreasing exactly by 1 correspondingly to any change of $f(0, u, t)$ : since $f(0, u, t)$ may change at most $m=|b|_{o}$ times, there is enough room for $\gamma_{i}(x, u, t)$ to decrease accordingly.

This concludes the proof of the theorem.

Conjecture 15 of [2] states that for every finite $n \geq 1$, the family of all $\Sigma_{n}^{-1}$ sets has positive undecidable numberings. This conjecture was verified in [9]. We are now in a position to extend it to all ordinal notations of nonzero ordinals.

Corollary 2.8. For every ordinal notation $a$, with $|a|_{0} \geq 1$, the family of all $\Sigma_{a}^{-1}$ sets has positive undecidable numberings.

Proof. By Theorem 2.1 and Theorem 2.2.

\section{Families without Friedberg numberings but with positive numberings}

We now show that at each level of the Ershov hierarchy there are infinite families without Friedberg numberings, but with positive numberings.

Theorem 3.1. For every ordinal notation $a, a>_{O} 1$, there exists an infinite family $\mathcal{A}$ such that $\operatorname{Com}_{a}^{-1}(\mathcal{A})$ has no Friedberg numberings but it has positive numberings.

Proof. We propose two different proofs of this result.

First proof. Let $a>_{O} 1$ be given. The first proof is an easy argument, following Ospichev's Theorem, [7], and the theorem of the previous section. 
Indeed, by [7], let $\mathcal{A}$ be an infinite family such that $\operatorname{Com}_{a}^{-1}(\mathcal{A}) \neq \emptyset$, but it contains no Friedberg numbering. Let $\mathcal{B}=\mathcal{A} \cup\{A\}$, where

$$
A= \begin{cases}\omega, & \text { if }|a|_{O} \text { is finite and odd; } \\ \emptyset, & \text { otherwise. }\end{cases}
$$

Then there is a numbering $\alpha \in \operatorname{Com}_{a}^{-1}(\mathcal{B})$ such that $\alpha(0)=A$. By Theorem $2.2 \mathrm{Com}_{a}^{-1}(\mathcal{B})$ has positive numberings (indeed a countable antichain), but no Friedberg numbering, since from a Friedberg numbering for $\mathcal{B}$ one could easily build one for $\mathcal{A}$.

Second proof. We show here that a slight modification of Ospichev's proof in [7] produces immediately a family without Friedberg numberings, but with a positive numbering. Let us fix a uniform effective listing $\left\{\nu_{e}\right\}_{e \in \omega}$ of all $\Sigma_{a}^{-1}$ numberings. We build a family $\mathcal{A}$ without Friedberg numberings, by building a positive numbering $\alpha$ of the family. We define $\alpha$ by defining $f(e, x, s)$ (i.e. $\alpha(e)(x)=\lim _{s} f(e, x, s)$ ) and a corresponding mind-change function $\gamma$. (We refer again here for notations and notions, to Definition 1.2).

The construction is by stages. At each subsequent stage, all parameters maintain the same values as at the previous stage, unless explicitly redefined.

Stage 0. Define $f(e, x, 0)=0, \gamma(e, x, 0)=\uparrow$, for all $e, x$.

Step 1. Define, for all $e$,

$$
f(2 e, 2 e+1,1)=f(2 e+1,2 e+1,1)=1
$$

and

$$
\gamma(2 e, 2 e+1,1)=\gamma(2 e+1,2 e+1,1)=1 .
$$

Since the values of each $\alpha(i)$ on odd numbers will never be redefined, notice that this will have the effect that, for $i \neq j$,

$$
\alpha(i)=\alpha(j) \Rightarrow\{i, j\}=\{2 e, 2 e+1\}, \text { some } e .
$$

Define also

$$
f(2 e, 2(2 e), 1)=f(2 e+1,2(2 e+1), 1)=1
$$

and

$$
\gamma(2 e, 2(2 e), 1)=\gamma(2 e+1,2(2 e+1), 1)=2 .
$$

Stage $s>1$. Consider all $e \leq s$. If there are no $t<s$ and no distinct $i, j \leq s$ such that

$$
\nu_{e}(i, 2(2 e), t)=\nu_{e}(j, 2(2 e+1), t)=1,
$$

but, at stage $s$,

$$
\nu_{e}(i, 2(2 e), s)=\nu_{e}(j, 2(2 e+1), s)=1,
$$


then define

$$
f(2 e, 2(2 e), s)=f(2 e+1,2(2 e+1), s)=0,
$$

(these values will never be redefined) and

$$
\gamma(2 e, 2(2 e), s)=\gamma(2 e+1,2(2 e+1), s)=1 .
$$

Might be I am wrong, but it seems to me that the actions above are incomplete: the opponent can do empty both sets $\nu_{e}(i)$ and $\nu_{e}(j)$ at some moment, and later rebuild what he wish in $\nu_{e}(i)$ and $\nu_{e}(j)$. Moreover, he can build, also later, a copy of $\alpha(i)$ at a fresh index. Please, check your construction and proof thoroughly.

Moreover, for all $e^{\prime} \neq 2 e, 2 e+1$, let

$$
\begin{aligned}
& f\left(e^{\prime}, 2(2 e), s\right)=1-\nu_{e}(i, 2(2 e), s) \\
& f\left(e^{\prime}, 2(2 e+1), s\right)=1-\nu_{e}(j, 2(2 e+1), s)
\end{aligned}
$$

and

$$
\begin{aligned}
& \gamma\left(e^{\prime}, 2(2 e), s\right)=\gamma_{e}(i, 2(2 e), s) \\
& \gamma\left(e^{\prime}, 2(2 e+1), s\right)=\gamma_{e}(j, 2(2 e+1), s)
\end{aligned}
$$

where $\gamma_{e}$ is a mind-change function corresponding to $\nu_{e}$.

Verification. We follow closely [7]. If $\mathcal{A}$ is the family numerated by $\alpha$, and we never find a stage $s$ and distinct numbers $i, j$ such that $\nu_{e}(i, 2(2 e), s)=$ $\nu_{e}(j, 2(2 e+1), s)=1$, then the family enumerated by $\nu_{e}$ does not contain distinct sets containing $2(2 e), 2(2 e+1)$, thus $\nu_{e}$ does not enumerate $\mathcal{A}$. Otherwise, there are two cases to consider:

1. $2(2 e) \in \nu_{e}(i)$ or $2(2 e+1) \in \nu_{e}(j)$ : again $\nu_{e}$ does not enumerate $\mathcal{A}$ since no set in $\mathcal{A}$ contains either element;

2. $2(2 e) \notin \nu_{e}(i)$ and $2(2 e+1) \notin \nu_{e}(j)$ : then if $\nu_{e}$ numbers $\mathcal{A}$ we have that $i, j$ are distinct indices of $\alpha(2 e)=\alpha(2 e+1)$, since $\nu_{e}(i)$ can only be $\alpha(2 e)$ and $\nu_{e}(j)$ can only be $\alpha(2 e+1)$.

It remains to show that $\alpha$ is positive. Notice that, for distinct $i, j$, equation (3.1) holds, and consequently we have:

$$
\begin{aligned}
& \alpha(2 e)=\alpha(2 e+1) \Leftrightarrow \\
& \quad(\exists s)(\exists i, j)\left[i \neq j \text { and } \nu_{e}(i, 2(2 e), s)=\nu_{e}(j, 2(2 e+1), s)=1\right] .
\end{aligned}
$$

Question 17 of [2] asks whether, for any $n \geq 1$, families of $\Sigma_{n}^{-1}$ sets with positive numberings have also decidable numberings. We show in fact that this is not so for every level (finite or infinite) of the Ershov hierarchy:

Corollary 3.2. For every ordinal notation a of a nonzero computable ordinal, there exists a family $\mathcal{A}$ such that $\operatorname{Com}_{a}^{-1}(\mathcal{A})$ has no decidable numberings but it has infinitely many positive numberings, whose Rogers degrees form an antichain.

Proof. By Lemma 1.4, and the first proof of Theorem 3.1. 


\section{References}

[1] S. Badaev. Positive enumerations. Sib. Mat. Zh., 18(3):483-496, 1977.

[2] S. Badaev and S. Goncharov. The theory of numberings: open problems. In P. A. Cholak, S. Lempp, M. Lerman, and R. A. Shore, editors, Computability Theory and its Applications, volume 257 of Contemporary Mathematics, pages 23-38. American Mathematical Society, Providence, 2000.

[3] Yu. L. Ershov. A hierarchy of sets, I. Algebra and Logic, 7:47-73, 1968.

[4] Yu. L. Ershov. A hierarchy of sets, II. Algebra and Logic, 7:15-47, 1968.

[5] Yu. L. Ershov. A hierarchy of sets, III. Algebra and Logic, 9:34-51, 1970.

[6] S. Goncharov and A. Sorbi. Generalized computable numerations and nontrivial Rogers semilattices. Algebra and Logic, 36(6):359-369, 1997.

[7] S. Ospichev. Computable family of $\Sigma_{a}^{-1}$-sets without Friedberg numberings. In 6th Conference on Computability in Eueope, CiE 2010, 6th Conference on Computability in Eueope, CiE 2010. Ponta Delgada, Azores, pages 311-315, 2010.

[8] H. Rogers, Jr. Theory of Recursive Functions and Effective Computability. McGraw-Hill, New York, 1967.

[9] Zh. T. Talasbaeva. Positive numberings of families of sets in the Ershov hierarchy. Algebra and Logic, 42(6):413-418, 2003. 